OPEN ACCESS

Edited by:

Heming Lu,

People's Hospital of Guangxi Zhuang

Autonomous Region, China

Reviewed by: Imran Petkar,

Guy's and St Thomas' NHS

Foundation Trust,

United Kingdom

Jared Shenson,

University of Texas MD Anderson

Cancer Center, United States

*Correspondence:

Chia-Hsuan Lai

chiahsuan7092@gmail.com

Specialty section:

This article was submitted to

Head and Neck Cancer,

a section of the journal

Frontiers in Oncology

Received: 06 August 2021 Accepted: 15 September 2021 Published: 29 September 2021

Citation:

Tsai Y-T, Chen W-C, Hsu C-M, Tsai M-S, Chang G-H, Lee $Y-C$, Huang $\mathrm{El}$, Fang $\mathrm{C}-\mathrm{C}$ and Lai $\mathrm{C}-\mathrm{H}$ (2021) Survival-Weighted Health

Profiles in Patients Treated for Advanced Oral Cavity

Squamous Cell Carcinoma.

Front. Oncol. 11:754412.

doi: 10.3389/fonc.2021.754412

\section{Survival-Weighted Health Profiles in Patients Treated for Advanced Oral Cavity Squamous Cell Carcinoma}

\author{
Yao-Te Tsai ${ }^{1}$, Wen-Cheng Chen ${ }^{2}$, Cheng-Ming Hsu ${ }^{1}$, Ming-Shao Tsai ${ }^{1}$, Geng-He Chang ${ }^{1}$, \\ Yi-Chan Lee ${ }^{3}$, Ethan I. Huang ${ }^{1}$, Chiung-Cheng Fang ${ }^{2}$ and Chia-Hsuan Lai ${ }^{2 *}$ \\ 1 Department of Otorhinolaryngology-Head and Neck Surgery, Chang Gung Memorial Hospital, Chiayi, Taiwan, \\ 2 Department of Radiation Oncology, Chang Gung Memorial Hospital, Chiayi, Taiwan, ${ }^{3}$ Department of Otorhinolaryngology- \\ Head and Neck Surgery, Chang Gung Memorial Hospital, Keelung, Taiwan
}

Objectives: For patients with oral cavity squamous cell carcinoma (OSCC), particularly for those with advanced disease, quality of life (QoL) is a key outcome measure. Therefore, we estimated survival-weighted psychometric scores (SWPS), life expectancy (LE), and quality-adjusted LE (QALE) in patients with advanced OSCC.

Methods and Materials: For estimation of survival function, we enrolled 2313 patients with advanced OSCC diagnosed between January 1, 2007, and December 31, 2013. The patients were followed until death or December 31, 2014. To acquire the QoL data, data from 194 patients were collected by employing the Taiwan Chinese versions of the Quality of Life Questionnaire Core 30 and Quality of Life Questionnaire Head and Neck 35 developed by the European Organisation for Research and Treatment of Cancer and the EQ-5D-3L between October 1, 2013, and December 31, 2017. The LE of the patients with OSCC were estimated through linear extrapolation of a logit-transformed curve. SWPS and QALE were determined by integrating the LE and corresponding QoL outcomes.

Results: For the patients with advanced OSCC, the estimated LE and QALE were 8.7 years and 7.7 quality-adjusted life years (QALYs), respectively. The loss of LE and QALE was 19.0 years and 20.0 QALYs, respectively. The estimated lifetime impairments of swallowing, speech, cognitive functioning, physical functioning, social functioning, and emotional functioning were $8.3,6.5,6.5,6.1,5.7$, and 5.4 years, respectively. The estimated lifetime problems regarding mouth opening, teeth, social eating, and social contact were 6.6, 6.1, 7.5, and 6.1 years, respectively. The duration of feeding tube dependency was estimated to be 1.6 years.

Conclusions: Patients with advanced OSCC had an estimated LE of 8.7 years and QALE of 7.7 QALYs. SWPS provided useful information regarding how advanced OSCC affects the subjective assessment of QoL. Our study results may serve as a reference for the allocation of cancer treatment resources.

Keywords: oral cavity squamous cell carcinoma, quality of life, survival-weighted psychometric scores, life expectancy, quality-adjusted life expectancy 


\section{INTRODUCTION}

Oral squamous cell carcinoma (OSCC) is the sixth most common cancer in the world, and its incidence has been increasing, with an annual incidence approaching 500,000 (1, 2). In Taiwan, because of the high prevalence of betel nut chewing and cigarette smoking (3), OSCC is the fourth most common cancer among men. Approximately $60 \%$ of patients with OSCC present with locoregionally advanced disease (stage III or IV) at diagnosis (1), and the 5-year survival rate is only $10 \%-40 \%$ (2). Both OSCC and its treatments can significantly impair patients' quality of life (QoL) and functional status. Conventionally, the outcome assessments for OSCC consider both physician and patient perspectives, with physicians objectively reporting survival, local control, and complication rates and patients subjectively reporting physical, emotional, social, and psychological outcomes (3). Patient reported outcomes are increasingly studied (4), and OSCC patients have reported varying degrees of physical problems [e.g., eating and speaking changes (5)], mental stress [e.g., fatigue, anxiety, and depression $(6,7)]$, and altered interpersonal relationships [e.g. social isolation, work impairment, and disrupted social relationships $(5,8)]$. Among head and neck cancer (HNC) patients, OSCC patients experience the worst QoL and function (9). Patients with advanced OSCC frequently experience moderate to severe QoL and functional impairments attributable to their extensive tumor invasion or multidisciplinary treatments, such as ablative surgery and radiation therapy $(9,10)$. Surgery plus adjuvant radiotherapy can result in more severe and prolonged QoL disturbance compared with radiotherapy alone (11), and psychosocial and functional impairment may persist for a long time $(5,8)$. Hence, periodic review of QoL and the use of questionnaires may facilitate communication between patients and physicians and thereby optimize cancer treatments and nutritional interventions, potentially improving survival in patients with OSCC (12).

The quality-adjusted life-expectancy (QALE) that considers both survival and QoL is widely applied for cancer patient care and clinical research $(13,14)$. Studies have compared and quantified QALE in patients with HNC by estimating life expectancy (LE) and quality-adjusted LE (QALE) $(14,15)$. However, these studies enrolled highly heterogeneous samples and did not consider survival-weighted psychometric scores (SWPS). In the present study, we investigated the feasibility of estimating QALE and SWPS by combining mean QoL scores at various intervals with survival function in patients with advanced OSCC.

\section{MATERIALS AND METHODS}

\section{Patients}

Figure 1 presents the study flowchart. Patients diagnosed as having OSCC between January 1, 2007, and December 31, 2013, were retrospectively analyzed for survival estimation. Eligibility criteria were the following: (1) aged 18 to 75 years; (2) had newly diagnosed locally advanced OSCC (stage III or IV); (3) underwent curative treatments; and (4) had an Eastern Cooperative Oncology Group performance status of 0 to 2. We excluded patients who (1) underwent palliative treatment; (2) had a history of any cancer; (3) whose OSCC had already metastasized at diagnosis; or (4) had another cancer in addition to OSCC. Finally, a cohort of 2313 patients with advanced OSCC diagnosed during the study period was enrolled from our cancer registry database. Patients with OSCC who underwent treatments and follow-up at our hospital from October 1, 2013, to December 31, 2017, were prospectively enrolled for QoL questionnaire completion. Informed consent was obtained from all participants, and the institutional review board of our hospital approved the study protocol (No. 102-2668B). This study was performed in compliance with the tenets of the Declaration of Helsinki.

\section{Treatment Protocol}

Each patient underwent a routine workup consisting of comprehensive history taking, physical examination, flexible fiberoptic laryngoscopy, plain chest radiography, abdominal sonography, and pretreatment computed tomography or magnetic resonance imaging of the head and neck. In addition, the computed tomography of chest will be arranged if there is any abnormal finding on the plain chest radiography, and the positron emission tomography/computed tomography scan will be performed in patients with stage IV disease or if there is any finding suspicious for metastasis in the aforementioned studies. All patients underwent either primary ablative surgery with adjuvant therapy or radiotherapy (RT)/chemoradiotherapy (CRT) with curative intent. The cancer staging manual of the American Joint Committee on Cancer (2010) was used for OSCC staging. The types of adjuvant therapy were determined by the tumor board conference according to institutional guidelines. The detailed adjuvant treatment guidelines in our institute and their comparison with the National Comprehensive Cancer Network guidelines have been reported by Lin et al. (16). In brief, patients diagnosed as having a pathologic T4 disease and single metastatic neck lymphadenopathy are provided adjuvant RT, whereas those diagnosed as having extranodal extension, multiple metastatic lymphadenopathies, or positive surgical margins are administered adjuvant CRT. If indicated, the intensity-modulated RT ( $2 \mathrm{~Gy} / \mathrm{d}, 5 \mathrm{~d} /$ week) was used to treat patients, and the radiation dose was 60-66 Gy in an adjuvant setting and 70-72 in a definitive setting. Platinum-based agents were used if chemotherapy was indicated. All patients were regularly followed up, at which time questionnaires were completed. Follow-up visits occurred during years 1-3 every three months and in years $4+$ every six months. At every followup visit, all patients were reviewed by speech-language pathologists and dietician and underwent complete physical examination including the fiberoptic laryngoscopy. Moreover, during the follow-up period, we executed head and neck magnetic resonance imaging or computed tomography at 6month intervals during the first 2 years and annually thereafter. 


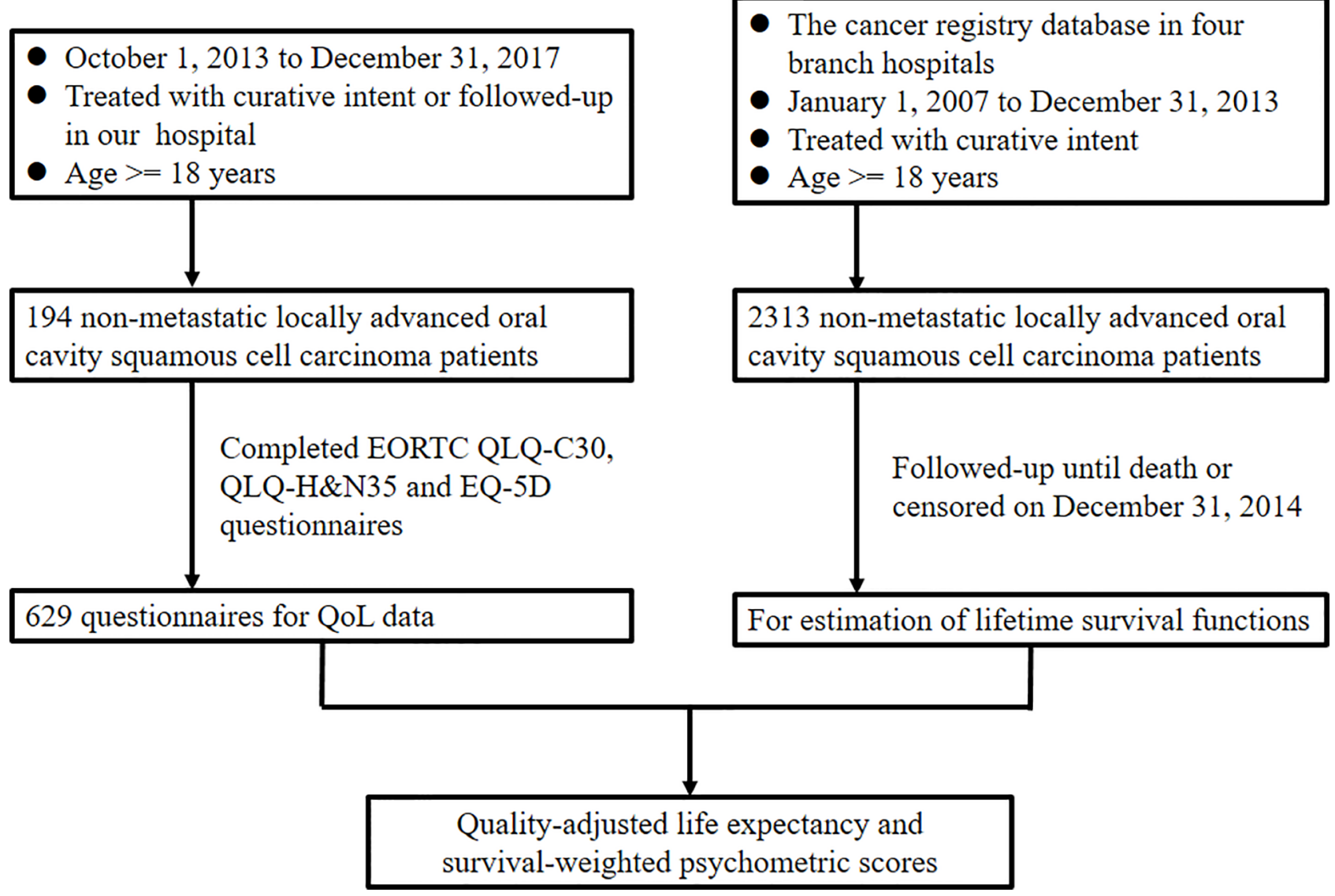

FIGURE 1 | Study design flowchart. EORTC, European Organisation for Research and Treatment of Cancer; H\&N, head and neck; QLQ, quality of life questionnaire; QoL, quality of life.

\section{QoL Instruments}

The Taiwan Chineseversions of the European Organisation for Research and Treatment of Cancer (EORTC) Quality of Life Questionnaire Core 30 (EORTC QLQ-C30) and EORTC Quality of Life Questionnaire Head and Neck 35 (EORTC QLQ-H\&N35) were used to assess QoL (17-19); these instruments, in translation and after cross-cultural adaptation to a Mandarinspeaking population, have been validated $(20,21)$. Per the EORTC scoring manual, for both instruments, scores were linearly transformed; all scales (multiple or single item) were scored 0-100 (22). A higher functioning score and QoL scale score indicated high functioning or QoL. By contrast, high scores on the symptom scales indicated more severe symptoms.

The EQ-5D-3L; Taiwanese version) was employed to assess general health and analyze cost utility (23). The EQ-5D-3L has five domains (pain/discomfort, mobility, anxiety/depression, self-care, and activities of daily living) and three levels of classification (no, some, and extreme problems). The health information derived from the five domains was transformed into health-related utility values by the time trade-off method (24). The utility value indicated the degree of general health status on a scale from 0 to 1 , with 0 representing death and 1 representing perfect health.

\section{Statistical Analysis}

Numbers with percentages were used for categorical variables, and means with standard deviations were used to indicate continuous variables. The survival duration of the 2313 patients from the cancer registration database was defined as the duration from the date of curative treatment to death or censoring on December 31, 2014. We then plotted the KaplanMeier curves for overall survival estimation. On the basis of the life table of the general population in Taiwan, the Monte Carlo method was applied to determine the survival function of the reference population (matched for age and sex) (25). Linear extrapolation of a logit-transformed curve of the survival ratio between patients with OSCC and the reference population was performed to obtain the LE of the patients with OSCC (25-27). Kernel smoothing of the QoL data from 194 patients was applied to estimate average QoL function (27). The functional disabilities or symptoms were plotted against time at the beginning of curative treatment. From then until the attainment of every QoL follow-up data point, the survival outcomes were combined with the psychometric scores or utility values to calculate the SWPS or QALE (3). In brief, the utility values or psychometric scores at different time points were multiplied with the 
corresponding lifetime survival probabilities over the course of cancer to obtain the quality-adjusted survival curve, of which the area under the curve would be the QALE or SWPS (28). The LE implies the expected total duration of living under a certain degree of unhealthy status after the treatments; the QALE can be interpreted as the expected total duration of living under a perfect healthy condition after the treatments. Each SWPS in a psychometric item can be interpreted as the expected total duration of living under a condition with a problem in that item after the treatments. The utility value was assumed to be 1 for the reference population during the study period. Hwang et al. proposed a minimum sample size of 50 for generating the mean QoL function curve (27). Considering the 7-year follow-up data and extrapolation to 50 years of survival, we estimated the LE, QALE, and SWPS of patients with OSCC. SPSS Statistics for Windows, version 17.0 (SPSS Inc., Chicago, IL, USA) was used for statistical analysis, and $\mathrm{p}<.05$ was considered to indicate statistical significance. Survival extrapolation was performed using iSQoL [http://sites.stat.sinica.edu/tw/isqol/; validated in $(13,29,30)$ ].

\section{RESULTS}

\section{Patient Characteristics}

Table 1 presents the patients' baseline characteristics. The survival data of 2313 patients with OSCC were used for lifetime survival estimates. Another 194 patients were selected for QoL questionnaire completion. Among the enrolled patients, the most common stage of OSCC was stage IVA $(n=1413,61.1 \%)$, followed by stage III $(n=602,26.0 \%)$ and stage IVB $(n=298,12.9 \%)$. Two thousand one hundred five (91\%) patients received ablative surgery as their primary treatment modality; 1088 (47\%) patients underwent postoperative adjuvant CRT, and 448 (19.4\%) patients received adjuvant RT. Given the presence of the unresectable T4b disease, significant underlying comorbidities [e.g. end-stage liver disease (31) and severely reduced ejection fraction (32)], and the patient's willingness, approximately $10 \%$ of patients underwent definitive RT/CRT as their primary treatment $(33,34)$. Table 2 presents the results of the 629 valid responses to the EORTC QLQC30 and QLQ-H\&N35 completed by 194 patients with OSCC, which were stratified by time periods: post-treatment $<1$ year, $1-3$ years, and $>3$ years.

\section{Survival Outcome, LE, and QALE}

Among the 2313 patients with OSCC, the 5-year overall survival rate was $54.2 \%$ (median follow-up: 31.4 months; range: $0.7-97.1$ months). The LE and QALE for the reference cohort in Taiwan is 27.7 years and 27.7 quality-adjusted life years (QALYs). In our cohort of patients with OSCC, the estimated LE and QALE was 8.7 years (95\% confidence interval [CI]: 6.3-14.8 years) and 7.7 QALYs (95\% CI: 5.5-13.1 QALYs), respectively (Figure 2); thus, the estimated loss was 19.0 years and 20.0 QALYs, respectively (Figures 3A, B, respectively).

\section{Symptoms and Impaired Function}

The median period between curative treatments and questionnaire completion was 1.7 months (range: 1-158.2 months). We estimated that patients with OSCC experienced pain and consumed painkillers for 4.9 and 2.0 years, respectively (Figure 4). Regarding functional disabilities, the durations of impairments in cognitive, physical, social, emotional, and role functioning were estimated to be 6.5 (95\% CI: 4.8-11.6), 6.1 (95\% CI: 4.6-11.5), 5.7 (95\% CI: 4.4-10.0), 5.4 (95\% CI: 4.1-10.0), and 2.8 (95\% CI: 2.0-4.9) years, respectively (Figure 5). The durations of impairments in swallowing, speech, taste, and smell were estimated to be 8.3 (95\% CI: 6.4-15.0), 6.5 (95\% CI: 4.9-11.7), 3.6 (95\% CI: 2.7-6.6), and 3.0 (95\% CI: 2.2-4.1) years, respectively (Figure 6). The patients experienced problems involving mouth opening, teeth, social eating, and social contact for an estimated 6.6 (95\% CI: 5.0-11.4), 6.1 (95\% CI: 4.5-11.0), 7.5 (95\% CI: 5.7-13.3), and 6.1 (95\% CI: 4.311.1) years, respectively (Figure 6). The estimated duration of tube feeding dependence was 1.6 (95\% CI: 1.1-2.8) years. In addition, the dynamic changes of the utility values and functional impairments (Figure 7) as well as different problems (Figure 8) were also demonstrated.

\section{Extrapolation Validity}

The model-extrapolated 8-year overall survival outcomes (using the initial 7-year follow-up data of 2313 patients) was compared with the survival outcomes measured using the Kaplan-Meier method. As shown in Figure 9, the observed survival data were highly consistent with the estimated survival curve. The mean \pm standard deviation of estimated survival among the patients with OSCC was $58.9 \pm 1.0$ months, indicating a relative bias of only $0.3 \%$ from the observed survival (59.1 \pm 0.8 months) at the end of the 8-year follow-up period.

\begin{tabular}{|c|c|c|}
\hline Variables & $\begin{array}{l}\text { OSCC patients } \\
\quad(n=2313)\end{array}$ & $\begin{array}{l}\text { Patients completed QoL } \\
\text { questionnaires }(n=194)\end{array}$ \\
\hline $\begin{array}{l}\text { Age at diagnosis } \\
\text { (years, mean } \pm S D \text { ) }\end{array}$ & $51.9 \pm 10.9$ & $52.4 \pm 9.8$ \\
\hline \multicolumn{3}{|l|}{ Gender } \\
\hline Male & 2147 (92.8\%) & 192 (99.0\%) \\
\hline Female & 166 (7.2\%) & $2(1.0 \%)$ \\
\hline \multicolumn{3}{|l|}{ Overall stage } \\
\hline III & 602 (26.0\%) & $56(28.9 \%)$ \\
\hline IVA & $1413(61.1 \%)$ & 113 (58.2\%) \\
\hline IVB & 298 (12.9\%) & 25 (12.9\%) \\
\hline \multicolumn{3}{|l|}{ T classification } \\
\hline $\mathrm{T} 1$ & 124 (5.4\%) & $16(8.2 \%)$ \\
\hline T2 & 496 (21.4\%) & 46 (23.7\%) \\
\hline Т3 & 428 (18.5\%) & 23 (11.9\%) \\
\hline T4A & 988 (42.7\%) & 84 (43.3\%) \\
\hline T4B & 277 (12.0\%) & 25 (12.9\%) \\
\hline \multicolumn{3}{|l|}{ N classification } \\
\hline NO & 870 (37.6\%) & 105 (54.1\%) \\
\hline $\mathrm{N} 1$ & $514(22.2 \%)$ & 28 (14.5\%) \\
\hline N2 & 918 (39.7\%) & 60 (30.9\%) \\
\hline N3 & $11(0.5 \%)$ & $1(0.5 \%)$ \\
\hline \multicolumn{3}{|l|}{ Curative treatment } \\
\hline Surgery & 2105 (91.0\%) & $174(89.7 \%)$ \\
\hline Adjuvant CRT & 1088 (47.0\%) & 123 (63.4\%) \\
\hline Adjuvant RT & 448 (19.4\%) & $51(26.3 \%)$ \\
\hline Curative RT/CRT & 208 (9.0\%) & 20 (10.3\%) \\
\hline
\end{tabular}

CRT, chemoradiotherapy; QoL, quality of life; OSCC, oral cavity squamous cell carcinoma; $R T$, radiotherapy; $S D$, standard deviation. 
TABLE 2 | The mean scores of the EORTC QOL scales in different periods of time.

\begin{tabular}{|c|c|c|c|}
\hline & T1 scores $( \pm \mathrm{SD})$ & T2 scores ( \pm SD) & T3 scores ( \pm SD) \\
\hline \multicolumn{4}{|l|}{ EORTC QLQ-30 } \\
\hline Global quality of life & $51( \pm 22)$ & $58( \pm 20)$ & $60( \pm 22)$ \\
\hline Physical functioning & $73( \pm 24)$ & $80( \pm 21)$ & $87( \pm 15)$ \\
\hline Emotional functioning & $72( \pm 26)$ & $78( \pm 23)$ & $79( \pm 24)$ \\
\hline Cognitive functioning & $78( \pm 24)$ & $75( \pm 20)$ & $77( \pm 19)$ \\
\hline Social functioning & $59( \pm 35)$ & $65( \pm 29)$ & $72( \pm 32)$ \\
\hline Role functioning & $75( \pm 34)$ & $80( \pm 31)$ & $88( \pm 23)$ \\
\hline Fatigue & $41( \pm 27)$ & $27( \pm 26)$ & $25( \pm 23)$ \\
\hline Nausea/vomiting & $10( \pm 19)$ & $03( \pm 13)$ & $04( \pm 11)$ \\
\hline Pain & $35( \pm 31)$ & $18( \pm 22)$ & $14( \pm 20)$ \\
\hline Dyspnea & $15( \pm 23)$ & $15( \pm 22)$ & $15( \pm 23)$ \\
\hline Insomnia & $35( \pm 36)$ & $24( \pm 30)$ & $26( \pm 30)$ \\
\hline Appetite loss & $28( \pm 32)$ & $13( \pm 22)$ & $11( \pm 19)$ \\
\hline Constipation & $17( \pm 22)$ & $17( \pm 27)$ & $12( \pm 19)$ \\
\hline Diarrhea & $13( \pm 23)$ & $7( \pm 15)$ & $10( \pm 17)$ \\
\hline Financial problems & $46( \pm 39)$ & $44( \pm 35)$ & $36( \pm 36)$ \\
\hline \multicolumn{4}{|l|}{ EORTC QLQ-H\&N35 } \\
\hline Pain & $29( \pm 26)$ & $18( \pm 24)$ & $12( \pm 14)$ \\
\hline Swallowing & $45( \pm 28)$ & $38( \pm 25)$ & $44( \pm 26)$ \\
\hline Senses (taste/smell) & $30( \pm 29)$ & $32( \pm 33)$ & $17( \pm 27)$ \\
\hline Speech & $34( \pm 29)$ & $29( \pm 28)$ & $35( \pm 30)$ \\
\hline Social eating & $48( \pm 30)$ & $42( \pm 31)$ & $47( \pm 34)$ \\
\hline Social contact & $26( \pm 27)$ & $22( \pm 25)$ & $27( \pm 28)$ \\
\hline Sexuality & $33( \pm 34)$ & $29( \pm 32)$ & $24( \pm 28)$ \\
\hline Teeth & $33( \pm 37)$ & $42( \pm 41)$ & $44( \pm 34)$ \\
\hline Opening mouth & $49( \pm 35)$ & $47( \pm 36)$ & $54( \pm 40)$ \\
\hline Dry mouth & $48( \pm 37)$ & $53( \pm 35)$ & $48( \pm 34)$ \\
\hline Sticky saliva & $50( \pm 35)$ & $34( \pm 33)$ & $33( \pm 35)$ \\
\hline Coughing & $33( \pm 30)$ & $31( \pm 23)$ & $26( \pm 25)$ \\
\hline Feeling ill & $45( \pm 35)$ & $26( \pm 26)$ & $21( \pm 25)$ \\
\hline
\end{tabular}

T1, within the first year after treatment beginning; T2, post-treatment 1-3 years; T3, post-treatment 3 years and thereafter.

EORTC, European Organization for Research and Treatment of Cancer; QLQ, quality of life; H\&N, head and neck; SD, standard deviation.

\section{DISCUSSION}

Patients with locally advanced OSCC tend to have more symptoms, more severe functional disabilities, and greater reductions in QoL due to aggressive tumor extension and metastatic lymphadenopathy necessitating extensive surgical

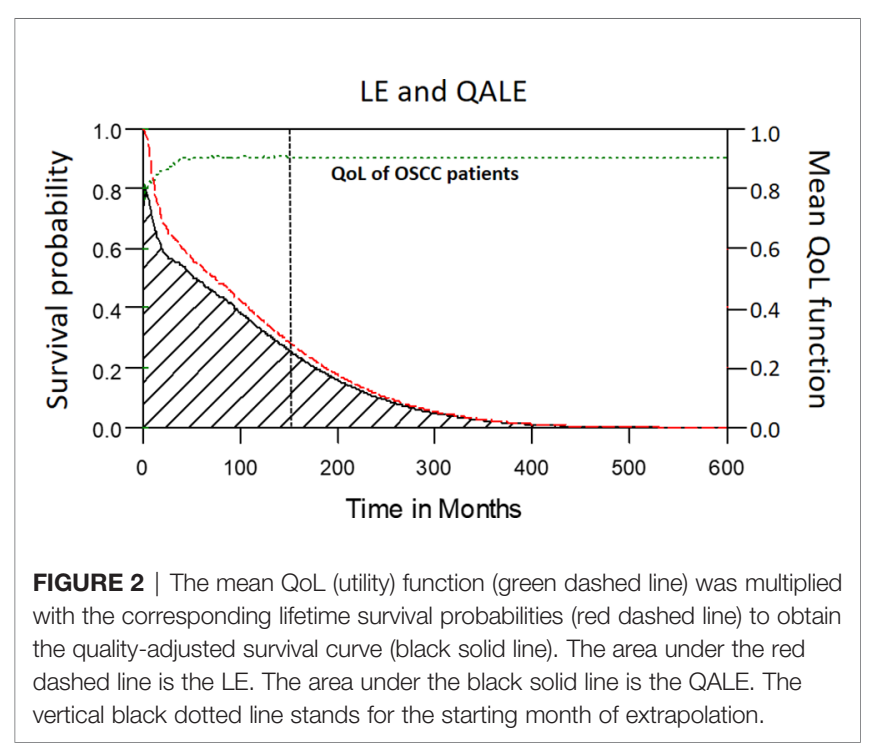

interventions or RT/CRT (4). Multidisciplinary management and advancements in treatment have facilitated the control of advanced OSCC (35); therefore, understanding the lifetime health burden of these patients is critical (36). To the best of our knowledge, this is the first study to describe QALE and lifetime symptoms or functional impairments in patients with locally advanced OSCC undergoing curative treatments. The estimated durations of problems concerning the teeth, mouth opening, social contact, and social eating all exceeded 6 years, consistent with results from a previous study (37). This may be explained by the significant lasting changes in oral structures, facial appearance, and social adaptation after curative treatment. We also observed that all the QoL domains excepting role function impairment ( 2.8 years), namely social, emotional, cognitive, and physical functioning, were adversely affected for over 5 years. This finding may be ascribable to the relatively young age of the patients at diagnosis, as well as to their active social participation and sufficient family support. The LE and QALE of the average patient with OSCC in Taiwan are approximately 12.2 years and 11.3 QALYs, respectively (15). Our estimates of LE and QALE, which were lower, are reasonable because we enrolled only patients with locally advanced disease. By integrating the QoL data from the QLQ-C30, QLQ-H\&N35, and EQ-5D-3L questionnaires with the survival function, we generated a multidimensional health profile from the patient perspective, enabling an intuitive understanding of the changes of QoL in patients with advanced 


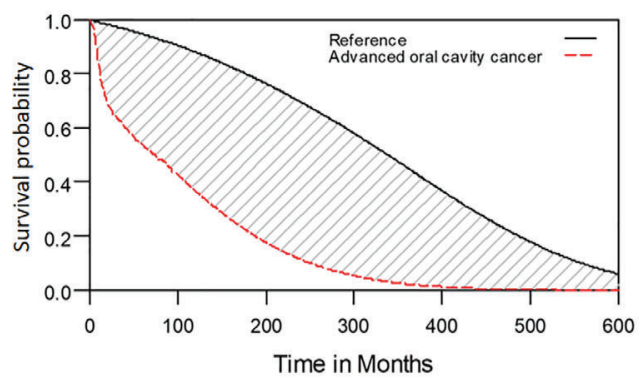

Estimated loss of QALE

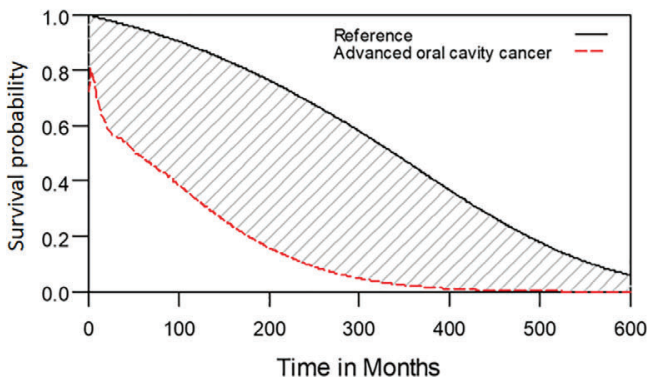

FIGURE 3 | Estimated loss of LE and QALE for advanced OSCC patients. (A) Estimated loss of LE; (B) Estimated loss of QALE.

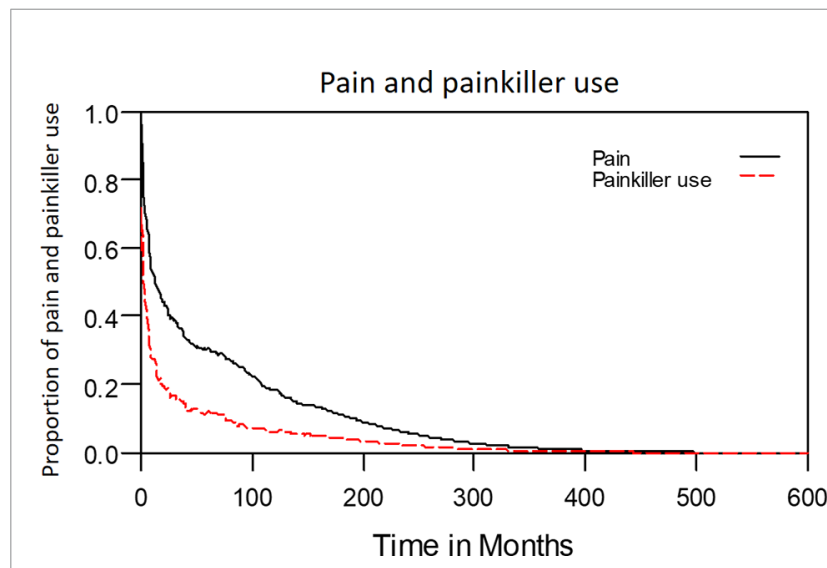

FIGURE 4 | Dynamic changes in pain and painkiller use in patients with locally advanced OSCC.

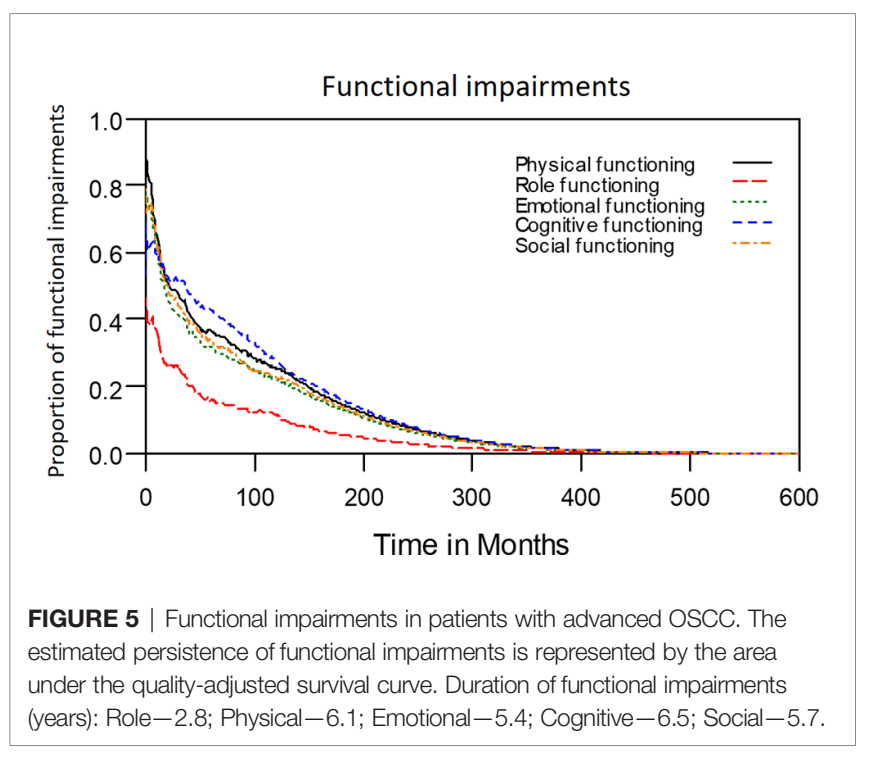

OSCC. Of note, OSCC and its treatments negatively impact patient QoL, particularly in those treated with ablative surgery (11). Extensive surgery considerably changes the facial appearance of patients with HNC, causing problems in social eating, swallowing, and speech and leading to social isolation and depression $(38,39)$. Given that $90 \%$ of our patients were treated with primary ablative surgery, their prolonged functional disabilities may be partially explained by the impacts of this extensive procedure. This highlights the necessity of developing psychosocial rehabilitation strategies for patients with advanced OSCC (40).

Our study results may extend the literature concerning QoL function in patients with locally advanced OSCC in several regards. First, because disease severity and treatment courses may influence various facets of QoL, a comprehensive assessment of psychometric scores for each QoL facet may yield a more holistic view. For instance, acute cancer- or treatment-related symptoms, such as pain and nausea or vomiting, may resolve gradually during follow-up. By contrast, patients with OSCC may experience prolonged physical distress and social functioning impairment that may or may not recover even after long-term disease remission is achieved (41). Multidimensional assessment may reflect the changes in QoL after the diagnosis of OSCC and may thus increase the feasibility of using QoL as an endpoint of treatment efficacy (42). Because the substantial change of the patient's QoL usually happened within the first 2 months after curative treatments due to the surgical morbidity, treatment related toxicity and its recovery, and most symptom burden tended to be stable after 1 year (43). Hence, we collected the QoL data more frequently in the first 2 months after treatments for better estimation of QALE or SWPS. In addition, the QoL after the last data collection time point is assumed to be the same thereafter. Accordingly, even $>50 \%$ of QoL data points came from within the first 2 months after treatments, it may cause little impact on the lifelong extrapolation. Second, patients' subjective judgments of QoL may change over time (44). Hence, we used the extrapolation method, which entails a simulation approach, for estimating the lifetime survival function. By integrating the extrapolated survival outcomes and the psychometric data, we acquired the 


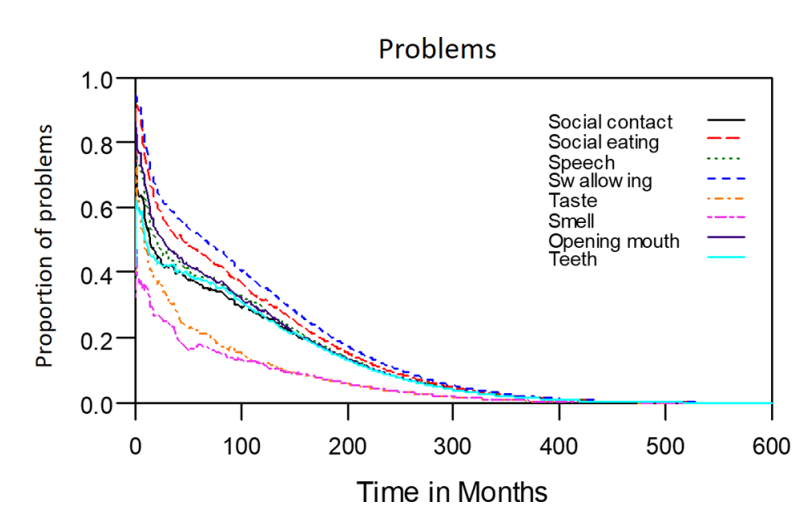

FIGURE 6 | Problems in patients with advanced OSCC. The estimated persistence of impairments or problems are represented by the area under the quality-adjusted survival curve. Duration of functional impairments or problems (years): Taste-3.6; Smell-3.0; Speech-6.5; Swallow-8.3. Problem-years endured: Open mouth-6.6; Dentition-6.1; Social eating7.5; Social contact-6.1.
SWPS for lifetime QoL assessments in patients with advanced OSCC. Changes in QoL scores over time correspond to the cancer treatment courses and disease severity in patients with cancer, and the QoL profile is particularly informative regarding emotional distress, physical performance, and social function (45); these findings accord with ours. Overall, SWPS may constitute a comprehensive approach for determining the lifetime QoL function of patients with advanced OSCC.

The health costs and economic burden of OSCC are comparable with or higher than those of other cancers (46). Despite their poorer survival outcomes, the patients with advanced OSCC used more resources (corresponding to higher expenditures) than did those with early-stage OSCC. This is attributable to the need of this patient group for multidisciplinary treatment, supportive care, and palliative care following repeated relapse (47). The results of this study demonstrated that compared with the reference population, patients with locally advanced OSCC had substantial losses of LE (19.0 years) and QALE (20.0 QALYs). Given that the QALY metric is commonly used to assess value in health care decision-
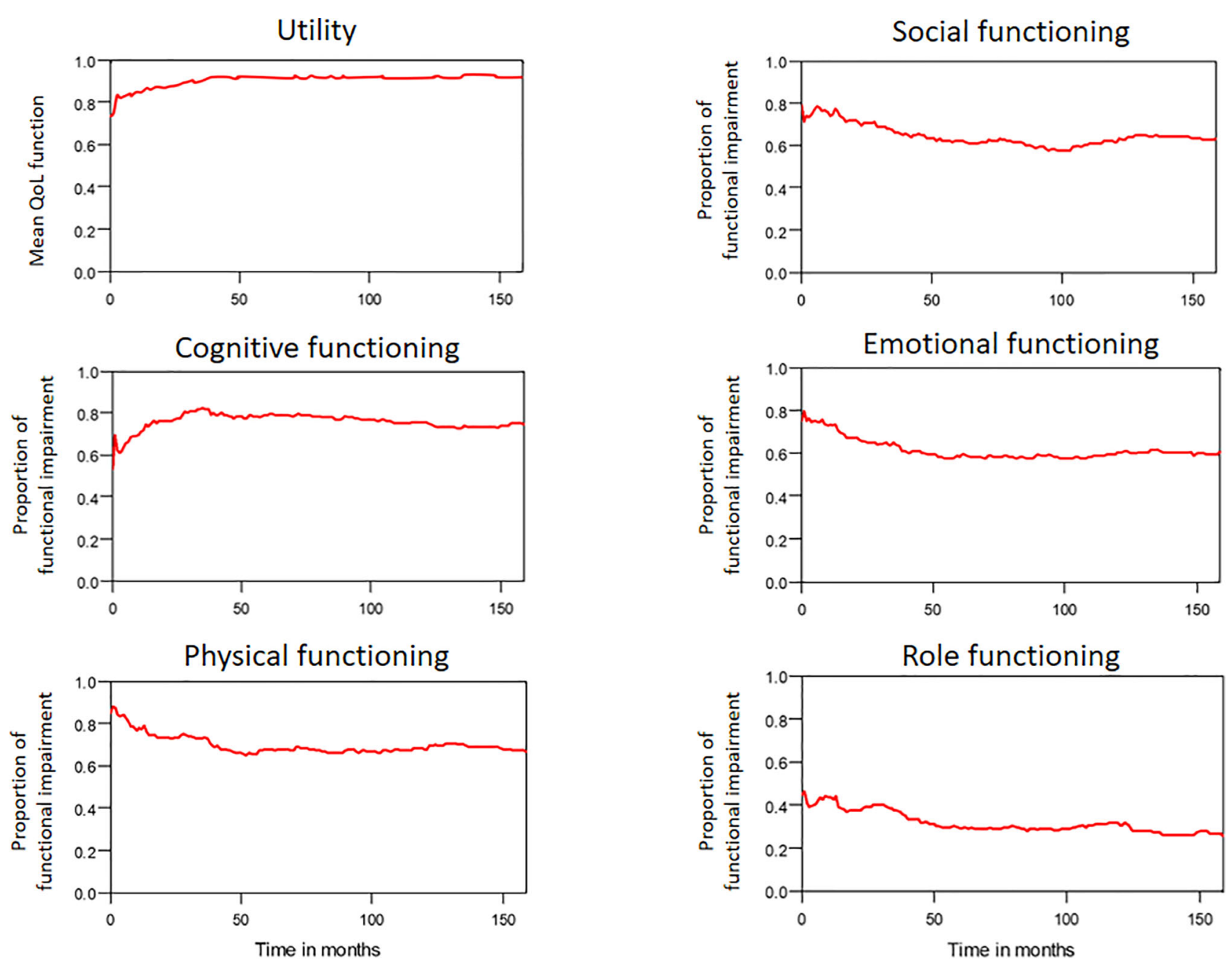

FIGURE 7 | The trends of mean QoL (utility) function and functional impairments in patients with OSCC. 

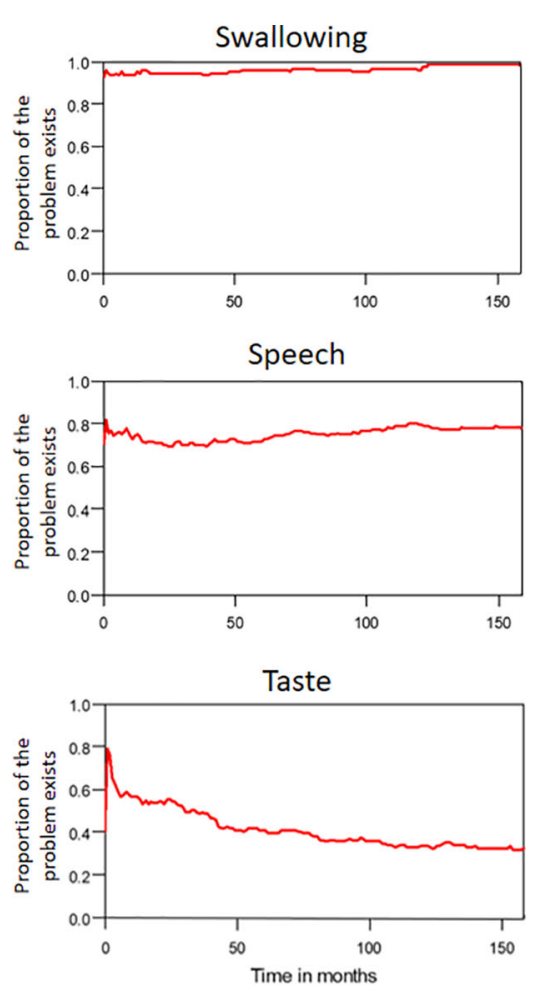
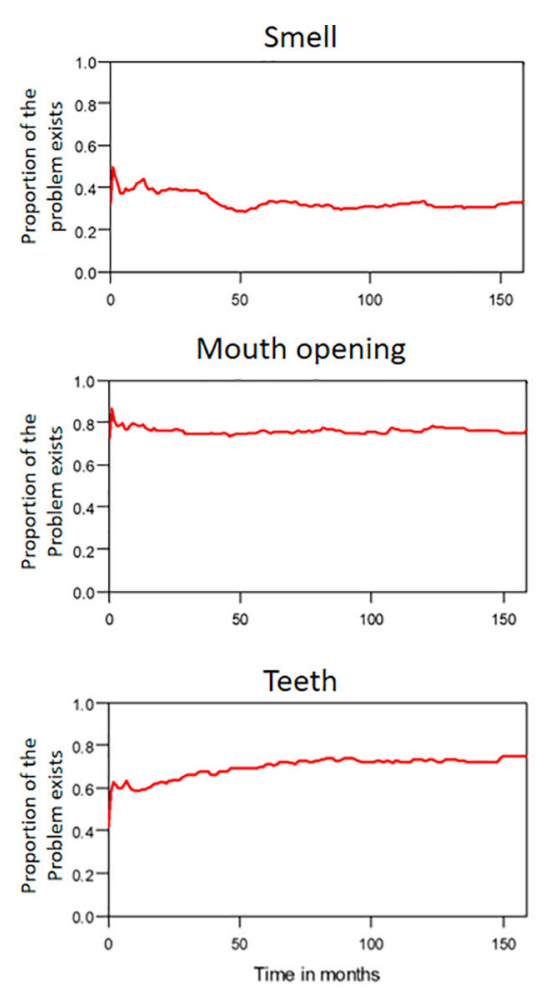

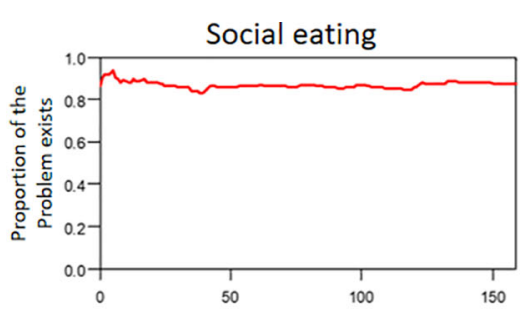

Social contact
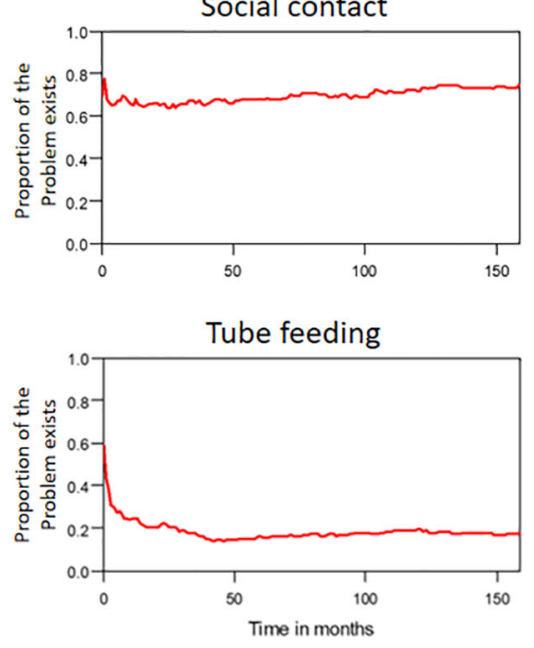

FIGURE 8 | The trends of different problems in patients with OSCC.

making (48), our data could yield useful information about resource allocation in advanced OSCC care.

This study has several limitations. First, the QALE and SWPS may have been overestimated for the following reasons. During extrapolation, the assumption of a constant level of QoL near the end of follow-up may have been distorted because real QoL usually declines with age (49). Moreover, patients who survived longer might have had a better QoL and completed more questionnaires (50). Further studies involving the administration of long-term QoL

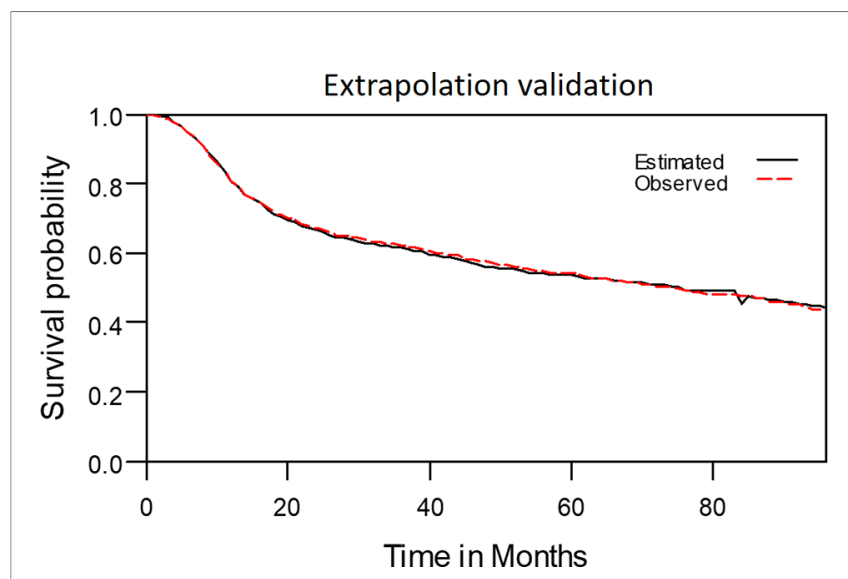

FIGURE 9 | The observed 8-year survival curve and the estimated 8-year survival curve matched properly. questionnaires and longer follow-up periods are warranted to confirm our findings. Second, the reference population utility was assumed to be 1 for the survival duration. Therefore, the loss of QALE among patients with OSCC may have been overestimated. Notably, Chung et al. indicated that women had an older mean age at diagnosis, less LE reduction, and a longer estimated QALE than men (15). However, our cohort had only two women with OSCC who completed the QoL questionnaires. Given that the computed tomography of chest was not routinely performed during staging workup in this study, small lung metastasis may have been underestimated and could negatively impact the patient's prognosis and QoL (51). This potential confounding factor may need to be considered in the results interpretation. Another potential confounding factor is that the improvement of the surgeon's technique and experience may lead to the better survival and QoL outcomes in patients who were treated in the later period of this study. Although our results involve intuitive assessment and appear reasonable, their interpretation should be made with these limitations in mind.

In conclusion, patients with advanced OSCC had an estimated LE and QALE of 8.7 years and 7.7 QALYs, respectively, and estimated LE and QALE losses of 19.0 years and 20.0 QALYs, respectively. The data on SWPS indicated that patients experienced multiple ongoing problems and functional disabilities over a long period of time following curative treatments. Future studies should evaluate whether information obtained from data on QALE and SWPS can be used to allocate health care resources and assess the 
impacts of surgery with different neoadjuvant or adjuvant protocols in patients with OSCC.

\section{DATA AVAILABILITY STATEMENT}

The raw data supporting the conclusions of this article will be made available by the authors, without undue reservation.

\section{ETHICS STATEMENT}

The studies involving human participants were reviewed and approved by Institutional review board of Chang Gung Memorial Hospital (No. 102-2668B). The patients/participants provided their written informed consent to participate in this study.

\section{REFERENCES}

1. Vokes EE, Weichselbaum RR, Lippman SM, Hong WK. Head and Neck Cancer. N Engl J Med (1993) 328(3):184-94. doi: 10.1056/NEJM199301213280306

2. Onizawa K, Nishihara K, Yamagata K, Yusa H, Yanagawa T, Yoshida H. Factors Associated With Diagnostic Delay of Oral Squamous Cell Carcinoma. Oral Oncol (2003) 39(8):781-8. doi: 10.1016/s1368-8375(03)00075-7

3. Lai CH, Chen WC, Fang CC, Chen MF. Survival-Weighted Health Profiles in Nasopharyngeal Cancer Patients. Front Oncol (2021) 11:635667. doi: 10.3389/ fonc.2021.635667

4. Abbas S, Tariq MUU, Raheem A, Saeed J, Hashmi SS, Karim M, et al. Assessment of Factors Affecting Quality of Life in Oral Squamous Cell Carcinoma Patients Using University of Washington Quality of Life Questionnaire. Cureus (2019) 11(1):e3904. doi: 10.7759/cureus.3904

5. List MA, Ritter-Sterr CA, Baker TM, Colangelo LA, Matz G, Pauloski BR, et al. Longitudinal Assessment of Quality of Life in Laryngeal Cancer Patients. Head Neck (1996) 18(1):1-10. doi: 10.1002/(SICI) 1097-0347(199601/02) 18:1<1::AID-HED1>3.0.CO;2-7

6. Neilson KA, Pollard AC, Boonzaier AM, Corry J, Castle DJ, Mead KR, et al. Psychological Distress (Depression and Anxiety) in People With Head and Neck Cancers. Med J Aust (2010) 193(S5):S48-51. doi: 10.5694/j.13265377.2010.tb03928.x

7. Sawada NO, de Paula JM, Sonobe HM, Zago MM, Guerrero GP, Nicolussi AC. Depression, Fatigue, and Health-Related Quality of Life in Head and Neck Cancer Patients: A Prospective Pilot Study. Support Care Cancer (2012) 20(11):2705-11. doi: 10.1007/s00520-012-1390-2

8. Penedo FJ, Traeger L, Benedict C, Thomas G, Dahn JR, Krause MH, et al. Perceived Social Support as a Predictor of Disease-Specific Quality of Life in Head-and-Neck Cancer Patients. J Support Oncol (2012) 10(3):119-23. doi: 10.1016/j.suponc.2011.09.002

9. Borggreven PA, Verdonck-de Leeuw IM, Muller MJ, Heiligers ML, de Bree R, Aaronson NK, et al. Quality of Life and Functional Status in Patients With Cancer of the Oral Cavity and Oropharynx: Pretreatment Values of a Prospective Study. Eur Arch Otorhinolaryngol (2007) 264(6):651-7. doi: 10.1007/s00405-007-0249-5

10. Rathmell AJ, Ash DV, Howes M, Nicholls J. Assessing Quality of Life in Patients Treated for Advanced Head and Neck Cancer. Clin Oncol ( $R$ Coll Radiol) (1991) 3(1):10-6. doi: 10.1016/s0936-6555(05)81034-9

11. McDonough EM, Varvares MA, Dunphy FR, Dunleavy T, Dunphy CH, Boyd JH. Changes in Quality-of-Life Scores in a Population of Patients Treated for Squamous Cell Carcinoma of the Head and Neck. Head Neck (1996) 18(6):48793. doi: 10.1002/(SICI)1097-0347(199611/12)18:6<487::AID-HED1>3.0.CO;2-Z

12. de Oliveira Faria S, Howell D, Vamondes Kulcsar MA, Eluf-Neto J. Nutritional Outcomes in Head and Neck Cancer Patients: Is Intensive

\section{AUTHOR CONTRIBUTIONS}

$\mathrm{C}-\mathrm{HL}$ and $\mathrm{W}-\mathrm{CC}$ were involved in the conception and design. $\mathrm{M}-\mathrm{ST}, \mathrm{C}-\mathrm{MH}$, and G-HC were involved in the analysis and data interpretation. Y-TT and CHL drafted the manuscript. Y-CL, $\mathrm{C}-\mathrm{CF}$, and $\mathrm{EH}$ revised the manuscript critically for intellectual content. All authors contributed to the article and approved the submitted version.

\section{FUNDING}

This study was supported by the Chang Gung Memorial Hospital (grant numbers: CORPG6D0251-3).The funding source had no role in the design of this study and had no role during its execution, analyses, interpretation of the data, or decision to submit results.

Nutritional Care Worth It? Cancer Treat Res Commun (2020) 25:100233. doi: 10.1016/j.ctarc.2020.100233

13. Hsu C, Wang JD, Hwang JS, Tien HF, Chang SM, Cheng AL, et al. SurvivalWeighted Health Profile for Long-Term Survivors of Acute Myelogenous Leukemia. Qual Life Res (2003) 12(5):503-17. doi: 10.1023/a:1025043113819

14. Lai CH, Chen MF, Fang FM, Chen WC. Estimation of Life Expectancy and Quality-Adjusted Life Expectancy in Non-Metastatic Nasopharyngeal Cancer Patients Treated by Intensity-Modulated Radiotherapy With or Without Chemotherapy. Oral Oncol (2014) 50(7):646-50. doi: 10.1016/j. oraloncology.2014.03.011

15. Chung CH, Hu TH, Wang JD, Hwang JS. Estimation of Quality-Adjusted Life Expectancy of Patients With Oral Cancer: Integration of Lifetime Survival With Repeated Quality-of-Life Measurements. Value Health Reg Issues (2020) 21:59-65. doi: 10.1016/j.vhri.2019.07.005

16. Lin CY, Fan KH, Lee LY, Hsueh C, Yang LY, Ng SH, et al. Precision Adjuvant Therapy Based on Detailed Pathologic Risk Factors for Resected Oral Cavity Squamous Cell Carcinoma: Long-Term Outcome Comparison of Cgmh and Nccn Guidelines. Int J Radiat Oncol Biol Phys (2020) 106(5):916-25. doi: 10.1016/j.ijrobp.2019.08.058

17. Aaronson NK, Ahmedzai S, Bergman B, Bullinger M, Cull A, Duez NJ, et al. The European Organization for Research and Treatment of Cancer Qlq-C30: A Quality-of-Life Instrument for Use in International Clinical Trials in Oncology. J Natl Cancer Inst (1993) 85(5):365-76. doi: 10.1093/jnci/85.5.365

18. Bjordal K, Hammerlid E, Ahlner-Elmqvist M, de Graeff A, Boysen M, Evensen JF, et al. Quality of Life in Head and Neck Cancer Patients: Validation of the European Organization for Research and Treatment of Cancer Quality of Life QuestionnaireH\&N35. J Clin Oncol (1999) 17(3):1008-19. doi: 10.1200/JCO.1999.17.3.1008

19. Bjordal K, Kaasa S. Psychometric Validation of the Eortc Core Quality of Life Questionnaire, 30-Item Version and a Diagnosis-Specific Module for Head and Neck Cancer Patients. Acta Oncol (1992) 31(3):311-21. doi: 10.3109/ 02841869209108178

20. Chie WC, Hong RL, Lai CC, Ting LL, Hsu MM. Quality of Life in Patients of Nasopharyngeal Carcinoma: Validation of the Taiwan Chinese Version of the Eortc Qlq-C30 and the Eortc Qlq-H\&N35. Qual Life Res (2003) 12(1):93-8. doi: 10.1023/a:1022070220328

21. Yang Z, Meng Q, Luo J, Lu Q, Li X, Li G, et al. Development and Validation of the Simplified Chinese Version of Eortc Qlq-H\&N35 for Patients With Head and Neck Cancer. Support Care Cancer (2012) 20(7):1555-64. doi: 10.1007/ s00520-011-1247-0

22. King MT. The Interpretation of Scores From the Eortc Quality of Life Questionnaire Qlq-C30. Qual Life Res (1996) 5(6):555-67. doi: 10.1007/ BF00439229

23. Chang TJ, Tarn YH, Hsieh CL, Liou WS, Shaw JW, Chiou XG. Taiwanese Version of the Eq-5d: Validation in a Representative Sample of the Taiwanese 
Population. J Formos Med Assoc (2007) 106(12):1023-31. doi: 10.1016/S09296646(08)60078-9

24. Lee HY, Hung MC, Hu FC, Chang YY, Hsieh CL, Wang JD. Estimating Quality Weights for Eq-5d (Euroqol-5 Dimensions) Health States With the Time Trade-Off Method in Taiwan. J Formos Med Assoc (2013) 112(11):699706. doi: $10.1016 /$ j.jfma.2012.12.015

25. Hwang JS, Wang JD. Monte Carlo Estimation of Extrapolation of QualityAdjusted Survival for Follow-Up Studies. Stat Med (1999) 18(13):1627-40. doi: 10.1002/(sici)1097-0258(19990715)18:13<1627::aid-sim159>3.0.co;2-d

26. Hwang JS, Tsauo JY, Wang JD. Estimation of Expected Quality Adjusted Survival by Cross-Sectional Survey. Stat Med (1996) 15(1):93-102. doi: 10.1002/(SICI)1097-0258(19960115)15:1<93::AID-SIM155>3.0.CO;2-2

27. Hwang JS, Wang JD. Integrating Health Profile With Survival for Quality of Life Assessment. Qual Life Res (2004) 13(1):1-10; discussion 11-4. doi: 10.1023/B:QURE.0000015299.45623.38

28. Lai WW, Chung CH, Lin CN, Yang SC, Hwang JS, Wang JD. Qalys and Medical Costs Saved From Prevention of a Cancer: Analysis of Nation-Wide Real-World Data of Taiwan With Lifetime Horizon. J Formos Med Assoc (2021). doi: 10.1016/j.jfma.2021.04.023

29. Chu PC, Wang JD, Hwang JS, Chang YY. Estimation of Life Expectancy and the Expected Years of Life Lost in Patients With Major Cancers: Extrapolation of Survival Curves Under High-Censored Rates. Value Health (2008) 11 (7):1102-9. doi: 10.1111/j.1524-4733.2008.00350.x

30. Lee HY, Hwang JS, Jeng JS, Wang JD. Quality-Adjusted Life Expectancy (Qale) and Loss of Qale for Patients With Ischemic Stroke and Intracerebral Hemorrhage: A 13-Year Follow-Up. Stroke (2010) 41(4):739-44. doi: 10.1161/ STROKEAHA.109.573543

31. Kao HK, Guo LF, Cheng MH, Chen IH, Liao CT, Fang KH, et al. Predicting Postoperative Morbidity and Mortality by Model for Endstage Liver Disease Score for Patients With Head and Neck Cancer and Liver Cirrhosis. Head Neck (2011) 33(4):529-34. doi: 10.1002/hed.21486

32. Healy KO, Waksmonski CA, Altman RK, Stetson PD, Reyentovich A, Maurer MS. Perioperative Outcome and Long-Term Mortality for Heart Failure Patients Undergoing Intermediate- and High-Risk Noncardiac Surgery: Impact of Left Ventricular Ejection Fraction. Congest Heart Fail (2010) 16 (2):45-9. doi: 10.1111/j.1751-7133.2009.00130.x

33. Foster CC, Melotek JM, Brisson RJ, Seiwert TY, Cohen EEW, Stenson KM, et al. Definitive Chemoradiation for Locally-Advanced Oral Cavity Cancer: A 20-Year Experience. Oral Oncol (2018) 80:16-22. doi: 10.1016/ j.oraloncology.2018.03.008

34. Stenson KM, Kunnavakkam R, Cohen EE, Portugal LD, Blair E, Haraf DJ, et al. Chemoradiation for Patients With Advanced Oral Cavity Cancer. Laryngoscope (2010) 120(1):93-9. doi: 10.1002/lary.20716

35. Liao CT, Kang CJ, Lee LY, Hsueh C, Lin CY, Fan KH, et al. Association Between Multidisciplinary Team Care Approach and Survival Rates in Patients With Oral Cavity Squamous Cell Carcinoma. Head Neck (2016) 38 Suppl 1:E1544-53. doi: 10.1002/hed.24276

36. Wu TY, Chung CH, Lin CN, Hwang JS, Wang JD. Lifetime Risks, Loss of Life Expectancy, and Health Care Expenditures for 19 Types of Cancer in Taiwan. Clin Epidemiol (2018) 10:581-91. doi: 10.2147/CLEP.S155601

37. Nemeth D, Zaleczna L, Huremovic A, Engelmann J, Poeschl PW, Strasz M, et al. Importance of Chewing, Saliva, and Swallowing Function in Patients With Advanced Oral Cancer Undergoing Preoperative Chemoradiotherapy: A Prospective Study of Quality of Life. Int J Oral Maxillofac Surg (2017) 46 (10):1229-36. doi: 10.1016/j.ijom.2017.05.005

38. Bjordal K, Freng A, Thorvik J, Kaasa S. Patient Self-Reported and Clinician-Rated Quality of Life in Head and Neck Cancer Patients: A Cross-Sectional Study. Eur J Cancer B Oral Oncol (1995) 31B(4):235-41. doi: 10.1016/0964-1955(95)00010-f

39. Langius A, Bjorvell $\mathrm{H}$ and Lind MG. Functional Status and Coping in Patients With Oral and Pharyngeal Cancer Before and After Surgery. Head Neck (1994) 16(6):559-68. doi: 10.1002/hed.2880160611
40. Rapoport Y, Kreitler S, Chaitchik S, Algor R, Weissler K. Psychosocial Problems in Head-and-Neck Cancer Patients and Their Change With Time Since Diagnosis. Ann Oncol (1993) 4(1):69-73. doi: 10.1093/ oxfordjournals.annonc.a058365

41. Howren MB, Christensen AJ, Karnell LH, Funk GF. Psychological Factors Associated With Head and Neck Cancer Treatment and Survivorship: Evidence and Opportunities for Behavioral Medicine. J Consult Clin Psychol (2013) 81(2):299-317. doi: 10.1037/a0029940

42. Pourel N, Peiffert D, Lartigau E, Desandes E, Luporsi E, Conroy T. Quality of Life in Long-Term Survivors of Oropharynx Carcinoma. Int J Radiat Oncol Biol Phys (2002) 54(3):742-51. doi: 10.1016/s0360-3016(02)02959-0

43. de Graeff A, de Leeuw JR, Ros WJ, Hordijk GJ, Blijham GH, Winnubst JA. Long-Term Quality of Life of Patients With Head and Neck Cancer. Laryngoscope (2000) 110(1):98-106. doi: 10.1097/00005537-200001000-00018

44. Brazier JE, Dixon S, Ratcliffe J. The Role of Patient Preferences in CostEffectiveness Analysis: A Conflict of Values? Pharmacoeconomics (2009) 27 (9):705-12. doi: 10.2165/11314840-000000000-00000

45. Velikova G, Wright P, Smith AB, Stark D, Perren T, Brown J, et al. SelfReported Quality of Life of Individual Cancer Patients: Concordance of Results With Disease Course and Medical Records. J Clin Oncol (2001) 19 (7):2064-73. doi: 10.1200/JCO.2001.19.7.2064

46. Lang K, Menzin J, Earle CC, Jacobson J, Hsu MA. The Economic Cost of Squamous Cell Cancer of the Head and Neck: Findings From Linked SeerMedicare Data. Arch Otolaryngol Head Neck Surg (2004) 130(11):1269-75. doi: 10.1001/archotol.130.11.1269

47. Schernberg A, Sagaon-Teyssier L, Schwarzinger MGroup ES. Clinical and Economic Burden of Head and Neck Cancer: A Nationwide Retrospective Cohort Study From France. Clinicoecon Outcomes Res (2019) 11:441-51. doi: 10.2147/CEOR.S198312

48. Garau M, Shah KK, Mason AR, Wang Q, Towse A, Drummond MF. Using Qalys in Cancer: A Review of the Methodological Limitations. Pharmacoeconomics (2011) 29(8):673-85. doi: 10.2165/11588250-000000000-00000

49. Orpana HM, Ross N, Feeny D, McFarland B, Bernier J, Kaplan M. The Natural History of Health-Related Quality of Life: A 10-Year Cohort Study. Health Rep (2009) 20(1):29-35.

50. Fryback DG, Dunham NC, Palta M, Hanmer J, Buechner J, Cherepanov D, et al. Us Norms for Six Generic Health-Related Quality-of-Life Indexes From the National Health Measurement Study. Med Care (2007) 45(12):1162-70. doi: 10.1097/MLR.0b013e31814848f1

51. Langendijk JA, de Jong MA, Leemans CR, de Bree R, Smeele LE, Doornaert P, et al. Postoperative Radiotherapy in Squamous Cell Carcinoma of the Oral Cavity: The Importance of the Overall Treatment Time. Int J Radiat Oncol Biol Phys (2003) 57(3):693-700. doi: 10.1016/s0360-3016(03)00624-2

Conflict of Interest: The authors declare that the research was conducted in the absence of any commercial or financial relationships that could be construed as a potential conflict of interest.

Publisher's Note: All claims expressed in this article are solely those of the authors and do not necessarily represent those of their affiliated organizations, or those of the publisher, the editors and the reviewers. Any product that may be evaluated in this article, or claim that may be made by its manufacturer, is not guaranteed or endorsed by the publisher.

Copyright (๑) 2021 Tsai, Chen, Hsu, Tsai, Chang, Lee, Huang, Fang and Lai. This is an open-access article distributed under the terms of the Creative Commons Attribution License (CC BY). The use, distribution or reproduction in other forums is permitted, provided the original author(s) and the copyright owner(s) are credited and that the original publication in this journal is cited, in accordance with accepted academic practice. No use, distribution or reproduction is permitted which does not comply with these terms. 